\title{
"Hoy somos capaces de crecer": mujeres indígenas rarámuri y su construcción identitaria en la universidad
}

Norma Luz González Rodríguez ${ }^{1 *}$

1 Universidad Autónoma de Chihuahua, Chihuahua, México Orcid: 0000-0002-5182-4574

Correo electrónico: nlgonzalez@uach.mx recibido Julio de 2017 Aceptado marzo de 2018

\section{Resumen}

Después de abordar el marco de referencia, este artículo describe las experiencias de las mujeres rarámuri como universitarias. El objetivo es identificar y explicar los procesos de articulación que llevan a cabo estas mujeres entre la educación universitaria y la construcción o reconstrucción de una identidad étnica y de género. Mi hipótesis es que ellas redefinen y revaloran - al igual que hacen con su identidad étnica- su identidad de género mediante estas experiencias académicas. Llevé a cabo este trabajo de investigación usando como método la etnografía. Encontré que algunos programas de ayuda e instituciones becantes fomentan y perciben la imagen de las mujeres rarámuri como las responsables de recuperar sus tradiciones, pero también aprender una cultura específica dictada por las instituciones. Las mujeres rarámuri también son seres autónomos que interpretan y responden activamente a su situación, pues hacen una valoración de sus condiciones históricas de subordinación como mujeres, que ahora determinan las relaciones interétnicas en la universidad, y ello me lleva a concebirlas como feministas.

\section{"Today we are able to grow": Rarámuri indigenous women and their identity construction at university}

\begin{abstract}
After addressing the frame of reference, this article describes the experiences of Rarámuri women as university students, with the objective of identifying and explaining the ways in which these women articulate university education and the construction or reconstruction of an ethnic and gender identity. My hypothesis is that they redefine and revalue, as they do with their ethnic identity, their gender identity through these academic experiences. I carried out this research using ethnography as a method. I found that grant institutions and aid programs encourage and perceive the image of
\end{abstract}

\section{Palabras clave}

Mujeres Indígenas; Estudiantes universitarias; Minorías; Identidad; Feminismo

\section{Key words}

Indigenous Women; University Students; Minorities; Identity; Feminism 
Rarámuri women as responsible for recovering their traditions, but also of learning a specific culture dictated by institutions. Rarámuri women are also autonomous beings who actively interpret and respond to their situation, as they assess their historical conditions as women, which now determine interethnic relations in the university, and this leads me to conceive them as feminists.

\section{"Hoje somos capazes de crescer": mulheres indígenas rarámuri e a sua construção de identidade na faculdade}

\section{Resumo}

Palavras-chave

Mulheres indígenas; Estudantes universitários; Minorias; Identidade; Feminismo
1. El programa "Oportunidades" fue instaurado a finales de la década de 1990. Su finalidad es disminuir la pobreza por medio de la seguridad alimentaria y de las transferencias económicas asociadas con la asistencia escolar de los niños y las niñas (con un mayor incentivo para ellas) en el nivel escolar secundario: una estrategia para tratar de nivelar, además, las oportunidades entre mujeres y hombres.
Depois de abordar o quadro de referência, este artigo descreve as experiências das mulheres Rarámuri como estudantes universitárias, sendo o objetivo identificar e explicar os processos de articulação realizados por essas mulheres entre a educação universitária e a construção ou reconstrução de uma identidade étnica e de gênero. Minha hipótese é que eles redefinem e revalorizam, como fazem com sua identidade étnica, sua identidade de gênero através dessas experiências acadêmicas. Eu realizei este trabalho de pesquisa utilizando a etnografia como um método. Descobri que as instituições de concessão e os programas de ajuda incentivam e percebem a imagem das mulheres de Rarámuri como responsáveis pela recuperação de suas tradições, mas também para aprender uma cultura específica ditada pelas instituições. As mulheres Rarámuri também são seres autônomos que interpretam e respondem ativamente à sua situação, porque fazem uma avaliação de suas condições históricas de subordinação como mulheres, que agora determinam as relações interétnicas na universidade, o que me leva a pensa-las como feministas.

\section{Introducción}

En México, la población indígena enfrenta múltiples factores que obstaculizan su formación universitaria: las barreras culturales y económicas, así como la falta de oportunidades y la baja calidad de la educación en sus lugares de origen, que se ubican en territorios marginales. Esta situación se ve reflejada en las cifras: en el año 2000, los indígenas universitarios apenas representaban el 0,98\% de la población total indígena de México (Durin y García, 2011).

La marginación en la que se ven inmersos los grupos indígenas se debe a que la población mestiza ocupa una posición dominante respecto de ellos en la escala de poder de la organización social en México, al adueñarse de aparatos como el Estado e instituciones como la escuela, lo que convierte a las poblaciones indígenas en minorías. Por otro lado, esta situación comienza a cambiar gracias a las políticas compensatorias, mediante programas focalizados en niñas, como el programa "Oportunidades" ${ }^{1}$ del gobierno federal.

La población rarámuri o tarahumara, que ocupa nuestro estudio, es originaria de la Sierra Madre Occidental o Sierra Tarahumara, ubicada en el estado Chihuahua, al norte de México. Las mujeres rarámuri que participaron en el presente estudio son parte de la juventud indígena que comenzó a ingresar a las universidades hace una década, apoyada por distintos programas de ayuda, tanto nacionales como internacionales. 
Por el contrario a la época colonial, en la que los frailes colonizadores negaron a las mujeres rarámuri la educación formal por ser ellas las principales transmisoras de la lengua y los valores de su comunidad; en la actualidad, las mujeres rarámuri son vistas, por instituciones becantes y programas de ayuda, como las actoras sociales ideales para ser beneficiarias de programas de atención, debido a la preservación que hacen de sus valores étnicos, pues las nuevas políticas educativas para la educación indígena tratan de recuperar lenguas en peligro de extinción, como lo es la lengua rarámuri, hablada con mayor frecuencia por las mujeres rarámuri que por los hombres.

Sin embargo, estos programas de alfabetización en sí rara vez cumplen con el objetivo de mantener o revitalizar una lengua en peligro, y se enfrentan a obstáculos en su implementación, pues no se basan en las realidades sociolingüísticas y socioeconómicas de los hablantes y no han implicado a la comunidad en la creación y ejecución de los programas que contemplen factores como son la pobreza en la que se encuentra sumida la población rarámuri, así como la explotación de sus tierras por parte de la población mestiza y blanca, tanto nacional como internacional; así como los conflictos ocasionados por el narcotráfico que tiene lugar en la Sierra Tarahumara, todo lo cual provoca la migración de esta población indígena a la ciudad.

A pesar de las problemáticas que enfrenta, el pueblo rarámuri representaba en 2012 el 0,44\% de la población de la Universidad Autónoma de Chihuahua, con más representantes que nunca antes. Esto debido a la estrategia de la Universidad de visibilizar la diversidad cultural, que, como señalan Dietz y Mateos (2011), es el referente prototípico del feminismo. Su noción de "cuotas" de acceso al poder es una vez más retomada por la lucha multicultural por el reconocimiento de grupos de identidades diversificadas, aunque delimitadas y diferenciadas, para así generar un sistema altamente complejo de trato diferenciado de grupos minoritarios.

El objetivo de esta política de "acción afirmativa" es transmitido a las esferas académicas y educativas, y consiste en paliar la discriminación persistente debida a criterios de género, color de la piel, religión, etnicidad, etc., que las minorías sufren por medio de una política deliberada de "discriminación positiva" (Pincus, 1994, cit. en Dietz y Mateos, 2011). Es gracias a esta discriminación positiva que hombres y mujeres rarámuri se encuentran en la universidad, y sobre todo gracias a la liberación de las mujeres, sustentada en el feminismo, que ha impactado los ideales de las poblaciones indígenas y se ha incorporado a sus propias exigencias, tal y como podemos apreciar en las siguientes declaraciones de una gobernadora rarámuri:

Antes las mujeres no podían ir a estudiar, pero ahora sí, porque nos han enseñado cuál es nuestro derecho, nos han enseñado que la mujer tiene derecho a trabajar y no solo de estar en la casa. Nos lo enseñaron personas que salían de la comunidad, que traían mensajes y nos informaban, tanto hombres como mujeres. (Catalina, gobernadora en la Sierra Tarahumara; noviembre de 2012)

Las declaraciones antes mencionadas y otras que se mostrarán en el presente trabajo me han llevado a utilizar el feminismo como concepto para interpretar las estrategias y las razones que llevan a las mujeres rarámuri a la universidad.

\section{Minorías étnicas y su experiencia en la educación formal}

Resulta útil para mi investigación considerar la teoría ecológico-cultural de Ogbu (Ogbu y Simons, 1998), pues toma en cuenta la manera en que las minorías se convirtieron en tales y la forma en que son tratadas en términos de las políticas educativas, lo que el 
autor denominó "sistema"; así como también el modo en que estas minorías se perciben a sí mismas y responden a la escolaridad como consecuencia de dicho tratamiento.

Considero entonces que, al indagar en la forma en que las mujeres rarámuri de mi investigación se han construido como minorías, así como las percepciones que tienen de sí mismas y las respuestas que pueden dar a su tratamiento - en tanto que minorías étnicas en la universidad-, podré conocer a fondo sus experiencias como universitarias y su construcción identitaria.

Avanzando sobre las respuestas que dan específicamente las mujeres indígenas a su tratamiento como minorías en las aulas universitarias, algunos estudios (Gil, 2005; Chávez, 2008) han encontrado que dichas mujeres suelen hacer frente a las políticas de exclusión educativa "echando mano" de su orgullo identitario al considerarse pueblos originarios, con riqueza cultural, por lo que se preocupan por el uso de su lengua originaria y el conocimiento de su cultura, aunque en algunos casos esto provoca discriminación por parte de sus compañeros y profesores (Chávez, 2008).

Por otro lado, el estudiantado indígena enfrenta procesos de reconocimiento como diferentes a los "otros" hegemónicos (las mayorías). Esto deriva en la construcción de una identidad autocrítica generada en una reflexión constante de interpretaciones, contradicciones y dilemas sobre sí, como personas (Gil, 2005).

Cabe anotar que existe un precedente (García, 2010) en el estudio de la población rarámuri universitaria, aunque no desde una perspectiva de género y en un tiempo en el que, a falta de apoyo institucional, era común que la condición étnica no se exaltara, aunque sí se revalorara. Esto me permite plantear la hipótesis de que las mujeres rarámuri redefinen y revaloran, al igual que hacen con su identidad ética, su identidad de género mediante su experiencia como universitarias. Con respecto a la identidad de género, es importante no obviar el hecho de que en las sociedades organizadas sobre la base de distinciones de género, como es nuestro caso, todas las personas tienen una identidad de género, cada quien es, siente y sabe que es hombre, mujer u otra categoría asignada por la sociedad y/o autoasignada. En esta clasificación genérica entran en juego sentimientos, actitudes, modelos de identificación o de rechazo. De este modo, esta identidad tiene que ver con la imagen que las personas, de manera más o menos consciente, proyectan en el ámbito social en relación con la ideología de género dominante (Maqueira, 2001, en Martín, 2008; Lagarde, 2011); por ello creo que la identidad de género de las mujeres rarámuri se transformó en su contacto con la población mayoritaria en el espacio universitario.

\section{Feminismo y minorías}

Es necesario anotar que la principal dificultad de equiparar el feminismo con el "punto de vista de la mujer" es que demos por sentado que las mujeres somos todas iguales y que existe solo un punto de vista femenino, que correspondería a la categoría "mujer" con identidad sociológica propia (Moore, 2009). Por ello las feministas negras discutieron la subordinación femenina, dada la incidencia de factores como la raza, la clase, la religión, etc., que no habían sido contemplados por las feministas blancas (Eudine, 2011), cuyo error estriba en generalizar las experiencias propias sin tomar en cuenta la de las mujeres negras, por ejemplo, al afirmar que la familia es una fuente de opresión, ignorando que no lo es necesariamente para las mujeres negras, a las que históricamente se ha negado - por medio de la esclavitud y otras condiciones- la posibilidad de disfrutar de una familia propia (Carby, 2012). Lo mismo ha sucedido con otras minorías étnicas, como las indígenas en México. Por ello me pregunto: ¿qué papel juega la familia en la experiencia universitaria de las mujeres indígenas? 
El feminismo negro es fundamental por ser precedente del feminismo indígena - pieza clave en el desarrollo de este artículo- ya que este último es incipiente y ha sido examinado muy poco, pero es recuperado en notables trabajos de autoras como Hernández (2008) y Gargallo (2014), que dan cuenta de feministas indígenas que reivindican frente al Estado el derecho a la diferencia cultural, mientras que al interior de sus comunidades rechazan aquellos "usos y costumbres" que atentan contra su dignidad.

\section{Metodología}

Pertenezco al grupo social que en las relaciones interétnicas de México es dominante, es decir, soy mestiza. Y en este camino de entender mi posición social y la de las mujeres rarámuri en nuestras relaciones interétnicas, han sido distintos feminismos (indígena, negro y poscolonial) los que me han ayudado a entender la forma en que la raza, el género, la lengua, la clase social y otras variables interaccionan e interseccionan en complejos procesos, como puede ser la educación en el aula. Así que me asumo y posiciono como feminista poscolonial, al ser yo misma habitante de un país que sufrió la colonización, y cuyas relaciones interétnicas y de género son producto de dicha colonización y ahora neocolonización para con los pueblos indígenas.

He llevado a cabo este trabajo de investigación usando como método la etnografía por medio de la observación participante en las aulas de la Universidad Autónoma de Chihuahua y otros puntos de reunión formal e informal de siete mujeres rarámuri universitarias de entre 19 y 21 años, de las carreras de Ciencias Agrotecnológicas y Enfermería, que he seguido de cerca a partir del año 2011 y hasta el 2014 durante los semestres de agosto-diciembre de cada año académico.

También realicé entrevistas en profundidad a nueve mujeres rarámuri de diversas carreras que se encontraban en distintas etapas o situaciones de formación universitaria, tales como en pleno desarrollo de esta, su finalización exitosa o la deserción. Además, llevé a cabo dos grupos focales: el primero, en el año 2012, involucró a ocho mujeres rarámuri de distintas carreras universitarias; y el segundo, en el año 2013, involucró a cinco hombres rarámuri: tres de ellos universitarios y dos recién graduados, que opinaron sobre las percepciones que tienen acerca de sus compañeras rarámuri.

Asimismo, entrevisté a diez actores sociales involucrados con el desarrollo académico de estas mujeres: profesores, tutores, gestores de programas de apoyo, así como con una gobernadora rarámuri (máxima autoridad de una de sus comunidades de origen). Todos los nombres originales de las personas que han participado en esta investigación han sido sustituidos por seudónimos, con la intención de preservar su anonimato.

También realicé, durante los meses de noviembre y diciembre de 2014 y enero de 2015, un taller de estudios de género en el que participaron ocho mujeres rarámuri universitarias de entre 19 y 22 años. Mediante este taller llevé a cabo una evaluación diagnóstica acerca de los conocimientos que estas mujeres tienen sobre los conceptos que manejo en mi investigación, para exponer posteriormente algunos avances de mi tesis y retomarlos en un debate; finalizando con un ejercicio de escritura en el que ellas expresaron sus reflexiones acerca de su experiencias como universitarias y su formación identitaria. El objetivo de esta investigación es identificar y explicar los procesos de articulación que llevan a cabo las mujeres indígenas rarámuri entre la educación universitaria y la construcción o reconstrucción de una identidad étnica y de género. 
2. El PIAl fue creado en 1998 por la Federación de Empresariado Chihuahuense (FECHAC) $y$ apoyado por 30 instituciones más de diversa índole.
3. Este proyecto tiene un antecedente en un programa que formaba parte de un proyecto más amplio que, a nivel mundial, estaba "dirigido a estudiantes provenientes de grupos sociales marginados" (Chávez, 2008, p. 2), convocado por ANUIES y Fundación Ford.

\section{Quiénes y cómo: selección y etiquetaje de estudiantes indígenas que llegan a la universidad}

Hasta antes del año 2005 era difícil determinar si existían estudiantes rarámuri en la universidad, pues no se contaba con ninguna clase de indicadores ni registros y no era común que ingresaran a este espacio académico. Pero en 2005, el Programa Interinstitucional de Atención al Indígena (PIAI) ${ }^{2}$ comenzó a trabajar en la educación de la población indígena a nivel superior (García, 2010). Entre 2006 y 2007, el PIAI realizó varias gestiones para ampliar la cobertura educativa, y surgió en este contexto el Programa Universitario Indígena (PUI), cuya finalidad ha sido obtener los apoyos necesarios para que los indígenas pudieran concluir los estudios universitarios.

En el año 2010 se hicieron presentes las primeras generaciones de indígenas en universidades chihuahuenses, con una matriculación de 82 estudiantes en la Universidad Autónoma de Chihuahua y 28 estudiantes más distribuidos en otras nueve universidades (García, 2010), ya que en ese mismo año, en la Universidad Autónoma de Chihuahua (UACH) se creó el Programa de Apoyo a Estudiantes Indígenas (PAEI) con el financiamiento del Banco Mundial ${ }^{3}$ y la supervisión de la Asociación Nacional de Universidades e Instituciones de Educación Superior (ANUIES), con el objetivo apoyar académica y económicamente a la población con ascendencia indígena que desearan ingresar a la Universidad.

Por otro lado, el estudiantado rarámuri de la UACH suele recibir también ayuda de instituciones gubernamentales y civiles (fundaciones) que son coordinadas por el Programa Universitario Indígena (PUI), que también incluye instituciones académicas (la UACH es la más importante), y que funciona con fondos de la Fundación Christensen y triangula el manejo de recursos con la Fundación del Empresariado Chihuahuense Asociación Civil (FECHAC).

El PAEI inicia en cada ciclo escolar sus gestiones con la organización de cursos para preparar a los alumnos indígenas hacia el examen de admisión, tratando de "nivelar" sus conocimientos académicos con los de la mayoría mestiza que predomina en la UACH. Además, el PAEI gestiona cupos especiales para quienes no superan el examen de admisión. En algunos casos, el estudiantado indígena acepta ofertas para estudiar carreras muy distintas a las que deseaba, pues facultades como las de Medicina y Derecho no aceptan fácilmente a quien no ha superado la prueba de admisión debido a la alta demanda con la que cuentan; por lo tanto, la mayoría de los aspirantes indígenas no acceden a estas carreras, pues no han tenido la formación académica exigida debido a las carencias académicas causadas por su marginación social.

Una vez que la población indígena encuentra un lugar en la universidad, el PAEI les ayuda a gestionar distintas becas económicas y alimenticias, así como la condonación de la matrícula por parte de la UACH; también lleva el control y el registro de sus calificaciones y principales necesidades académicas. El cometido de este programa es nivelar las oportunidades de desarrollo académico (PAEI, 2010); para ello ofrece también asesoría extracurricular, talleres, seminarios y conferencias que contribuyen al mejoramiento académico, y apoyo en la enseñanza del manejo de las Tecnologías de la Información y la Comunicación (TIC) al alumnado indígena.

Como resultado de las gestiones antes mencionadas, en el año 2012 en la UACH se encontraban matriculados 112 estudiantes rarámuri del total de 25.039 estudiantes, que representan el $0,44 \%$ de la población estudiantil. Del total de la población rarámuri universitaria, 56 eran mujeres; esto es el 50\% de la población rarámuri de la universidad, pero apenas un $0,22 \%$ de toda la población universitaria (Datos proporcionados por 
el Programa de Atención a Estudiantes Indígenas PAEI-UACH el 12 de noviembre de 2012). Además, existen ciertos filtros que los estudiantes rarámuri deben enfrentar antes de ingresar a la $\mathrm{UACH}$, tal como narra una estudiante:

En el último semestre del bachillerato mi maestra me entrevistó con preguntas en torno a la comunidad, de sus fiestas, tradiciones y el papel que tiene mi familia. Luego participé como aspirante para formar parte del grupo de estudiantes universitarios indígenas de la $\mathrm{UACH}$. A partir de que formé parte del grupo nos empezaron a asignar o a establecer aspectos que tenemos que cumplir para ser parte del mismo. (Azucena, Chihuahua, noviembre de 2014)

Podemos percatarnos, gracias a estas declaraciones, de que existen ciertas exigencias que los estudiantes deben cumplir para ser categorizados como indígenas, a la vez que ser portadores de derechos, pero también regulados en su comportamiento, y así propiciar la formación de un grupo étnico, pues es por medio de los grupos que las personas son llevadas de forma individual a aceptar la identidad, la agencia, los intereses y la voluntad impuestos, sin que necesariamente sean sentidos y experimentados (Brubaker, 2002). Por ejemplo, Simona, una de las estudiantes rarámuri que aceptó ser becada y "etiquetada" como tal, no siente serlo, al pertenecer a una religión protestante, y por ello negarse a participar en algunos rituales rarámuri que tienen un precedente católico y cuya realización es fomentada por una de las fundaciones becantes.

En relación con esta grupalidad no sentida, Brubaker (2002) afirma que la retroalimentación debidamente encuadrada y codificada puede configurar la experiencia posterior y aumentar los niveles de grupalidad, pues podemos entender las dinámicas de formación de grupos como un proyecto social, cultural y político, que apunten a transformar las categorías.

Aunque en el caso del estudiantado rarámuri la retroalimentación a veces no es muy efectiva. Por ejemplo, durante un encuentro estudiantil organizado por una fundación becante, esta les pidió a los estudiantes que escenificaran el papel de dicha fundación en relación con su contribución del desarrollo académico. Un equipo de estudiantes satirizó las políticas de la fundación y su insistencia en que asistieran a los eventos con la vestimenta étnica para que los donantes de cheques se sintieran complacidos en relación con su ideal de la forma en la que se ve alguien indígena. Sin embargo, y a pesar de esta crítica, la fundación no realizó ningún cambio en sus políticas.

El hecho de que las instituciones dominantes que brindan apoyo al estudiantado indígena decidan los rasgos que sus miembros deben poseer para ser concebidos como tales puede ser denominado "etiquetación selectiva" (Nayaran, 2000, en Hernández, 2008), y generar lo que Castells (2001) denominó "identidad legitimadora”. El contenido de esta identidad legitimadora, en nuestro caso, no está aún definido ni determinado del todo, pues se amolda a las exigencias de los actores sociales dominantes, como son los donadores de cheques, directivos y profesores de las facultades, gestores, etc. Y pareciera que, algunas veces, los estudiantes indígenas se convierten en personajes folclóricos que están en la universidad para cumplir con una política de inclusión, pero otras veces lo están para adquirir las herramientas que les permitan "salvar" a sus comunidades de origen. Estas identificaciones corresponden más a las mujeres, que son reconocidas por los gestores y el profesorado como más participativas y responsables que los hombres, pero son en realidad más abiertas a reproducir su cultura al tiempo que adquieren nuevas experiencias, tal como señalan Azucena y Citlaly, dos alumnas rarámuri: "He ido construyendo y reconstruyendo mi identidad, a partir de talleres y seminarios y conferencias. En mi estructura social siempre nos encontramos aprendiendo y formando parte de nuestro entorno cultural" (Azucena, Chihuahua, noviembre de 2014). 
He conseguido muchos logros que no imaginaba, aún tengo muchos sueños por cumplir y agradezco a las personas que me han apoyado para estar donde estoy y también los talleres que me han hecho crecer más como mujer y salir adelante. (Citlaly, Chihuahua, noviembre de 2014)

Vemos entonces que las instituciones fomentan y perciben más la imagen de las mujeres rarámuri como las responsables de "aprender y formar parte de su entorno cultural"; es decir, de recuperar sus tradiciones, pero también de aprender una cultura específica y dictada por dichas instituciones, por medio de talleres y foros, con la finalidad de transformar a su cultura existente. Por otro lado, no todas las mujeres rarámuri están de acuerdo con la construcción de una identidad legitimadora entendida como aquella impuesta y definida por las instituciones dominantes, tal como me fue posible percibir durante el encuentro de estudiantes indígenas organizado por la fundación arriba mencionada. En él, algunas mujeres expresaron durante uno de los recesos lo cansadas que estaban de que les "enseñaran" a fortalecer su identidad indígena y de que les dijeran siempre quiénes eran ellas o cómo tenían que ser.

Pese a lo anterior, las mujeres rarámuri de mi investigación son más susceptibles a aceptar estas dinámicas y a obtener por ello mayor éxito académico, ya que, como afirman otros trabajos (Gibson, 1988, en Ponferrada, 2012; González, 2015), las mujeres pertenecientes a minorías étnicas suelen mostrar identidades más flexibles y favorables a traspasar las fronteras étnicas que sus homólogos varones, buscando un beneficio para sí mismas, pero también para sus comunidades (Abajo y Carrasco, 2004; Gil, 2005; Durin y García, 2011; González, 2015), y se les facilita reproducir discursos que para ellas parecen bien fundados, por ejemplo, el de sentir orgullo por su identidad.

\section{Ser una mujer rarámuri en el aula}

Para la gran mayoría de las mujeres rarámuri con las que trabajé, la Universidad ha significado el espacio de encuentro interétnico más importante: es la primera vez que abandonan su lugar de origen, la Sierra Tarahumara, donde su contacto con la cultura dominante suele ser a través de los medios de comunicación como la radio y la televisión, o mediante los contenidos de los programas escolares, que al iniciar la educación secundaria dejan de ser bilingües y adaptados a su cultura; también tienen un contacto interétnico gracias al comercio de algunos productos - como las telas que los mestizos les venden-, así como en la creación de proyectos de infraestructura como hospitales, vías de ferrocarril, etcétera.

Aunque para las mujeres rarámuri de mi investigación haber llegado hasta un aula universitaria es una oportunidad muy significativa, es poco común que ellas participen en clase, pues no se sienten en libertad de expresar su opinión abiertamente, al no ser consideradas como personas "expertas" frente al despliegue de conocimientos occidentalizados, pues su formación académica previa ha sido deficiente debido a la marginación social, como expresa Frida:

Me he sentido muy aparte, porque ellos (los estudiantes mestizos) ya vienen más preparados, hablan de un libro, de un autor y yo no tenía conocimiento de ello, me siento fuera de lugar. Le he echado muchas ganas para estar al nivel. Sí me han dicho comentarios discriminatorios pero no les hago caso. (Frida, noviembre de 2012)

Casualmente, un año después de escuchar estas declaraciones de Frida, corroboré sus comentarios acerca de la discriminación que ha sufrido a causa de su desconocimiento de la literatura occidental, pues un estudiante mestizo de su grupo académico me comentó: "Los estudiantes rarámuri qué hacen aquí, no saben nada, las dos chicas que están en mi clase nunca hablan" (Enrique, en una conversación casual durante el 
mes de noviembre de 2013). Es común que los estudiantes mestizos no interactúen con las estudiantes indígenas, y menos aún al formar equipos de trabajo, como pude observar, pues las rechazan por su condición étnica. Por ello, las vivencias de soledad de las jóvenes rarámuri que participaron en mi investigación es similar a la de algunos jóvenes pertenecientes a minorías étnicas en contextos como el catalán/español, donde Ponferrada (2007) da cuenta de que las mujeres pertenecientes a minorías étnicas son acompañadas únicamente por algunas jóvenes de la mayoría dominante, porque estas últimas tienen menor prestigio social en su contexto; son ellas las que brindan su amistad a chicas de origen minoritario para así no sentirse solas. Estas chicas actúan como verdaderas "acogedoras".

Solo pude vislumbrar uno de estos casos de chicas "acogedoras" en Irene, una mujer mestiza mucho mayor que sus compañeras también mestizas. Ella había estado muchos años alejada de la vida académica y se sentía desligada de este ambiente, por eso estableció una relación de amistad con Alejandra, que se acercaba a ella siempre explicándole que, al ser rarámuri, necesitaba ayuda. Sin embargo, es difícil encontrar otro caso como el de Irene, así que las mujeres rarámuri suelen entablar relaciones de amistad solo entre ellas, o vivir su experiencia universitaria en solitario. Este fue el caso de Selene, quien, aunque tuvo éxito académico, nunca tuvo amigos mestizos y al finalizar su carrera no asistió a la fiesta de graduación organizada por su facultad.

Esta falta de empatía que tiene el alumnado mestizo con el alumnado rarámuri es explicada por Azucena, una estudiante rarámuri, como resultado de las políticas de la Universidad:

La universidad no tiene una idea acerca de cómo tratarnos pues el hecho de que nos den apoyo tan solo por ser indígenas hace que los demás estudiantes nos perciban como estudiantes sin mérito propio, excepto el de pertenecer a un grupo étnico, como el caso de un compañero, que consiguió una beca en el extranjero solo porque un investigador estaba interesado en su cultura. (Azucena, noviembre de 2012)

Existen otras causas más para el etiquetaje de la población rarámuri como "estudiantes sin mérito", casi todas ocasionadas en la falta de comprensión. Por ejemplo, Ana fue vista por los docentes de la Universidad como un caso perdido; explicaban su falta de interés en el aula debido a su condición de indígena, pero nunca profundizaron acerca de ello. No sabían que ella había soñado con estudiar arquitectura, pero esa carrera no es ofertada por la Universidad Autónoma de Chihuahua, sino únicamente por universidades privadas que no participan en el PUI. Así que Ana, al no poder pagar dicha carrera, aceptó estudiar Agrotecnología en la $\mathrm{UACH}$, trayendo con ello la frustración de dedicarle tiempo a unos estudios que no deseaba realizar, o de mentirle a su madre respecto de su falta de asistencia a clases para no desilusionarla, todo lo cual propició que se sintiera como la mujer que no deseaba ser.

Por otro lado, muchas de las mujeres rarámuri que son matriculadas en carreras distintas a las que deseaban le dan un giro distinto a esta oportunidad, y llegan por méritos propios a adquirir experiencias que no imaginaron. Tal es el caso de Mary, a quien "el gran amor a su tierra la lleva a enfocarse en sus estudios, ella desea aprender tanto para replicarlo en su pueblo para que pueda haber desarrollo, eso la llevó a la Universidad Federal de Uberlandia, en Brasil" (Rivas, 2015, s/p) en un intercambio escolar en el que fue apoyada por su facultad y otras instituciones y fundaciones, en el que aprendió mucho acerca de los cultivos que puede usar en su comunidad de origen (Rivas, 2015), pero Mary no fue seleccionada por ser rarámuri, sino por su desempeño académico.

A pesar de estas muestras de mérito propio de mis sujetas de estudio, he visto que parte del profesorado se dirige a las jóvenes rarámuri como un grupo homogéneo, 
como también señala uno de sus tutores: "siempre se están refiriendo a los estudiantes rarámuri como alumnos de bajo desempeño, siendo que no siempre es así, y además pareciera que no hay alumnos mestizos con bajo rendimiento académico, cuando todos sabemos que sí" (Salvador, profesor, Chihuahua, diciembre de 2013).

Tal como apuntan Abajo y Carrasco, la retórica habitual es abusar cada vez más de una idea popular de cultura esencialista aduciendo la existencia de "valores culturales" de tipo comunitarista a la minoría, contrarios a la orientación individualista del aprendizaje escolar mayoritario (2011). Estos etiquetajes son percibidos en los discursos del profesorado en las aulas e inciden en las percepciones que las minorías tienen de su propia posición escolar y el prestigio individual (Ponferrada, 2007), pero siguiendo la propuesta de Ogbu y Simons (1998) podemos entender que el estudiantado de minorías étnicas también son seres autónomos que interpretan y responden activamente a su situación pues las mujeres rarámuri - para evaluar su situación de minorías en el aula - hacen una valoración de las condiciones históricas que ahora determinan las relaciones interétnicas en este espacio, tal como se ve reflejado en las siguientes declaraciones de Aura:

Todo empezó en la época de la colonización española, trajo un cambio social y cultural muy fuerte. Ahora el equilibrio de poder es muy injusto. Los chabochi (los mestizos) tienen el conocimiento, las armas y el poder para hacer lo que les dé la gana. Si no nos hubieran invadido nuestro ingenio sería exponencial, nos cortaron las alas, nos crearon como personas incapaces, con miedo. Hoy somos capaces de crecer, de hacer nuestro propio conocimiento. (Aura, Chihuahua, enero de 2015)

Estas declaraciones reflejan un ejercicio de feminismo poscolonial e indígena, una forma de quilting (tejer) en la que se usan fragmentos de memoria para hilar pasado y presente, para así interpretar las historias "ocultas" de mujeres que han sido marginadas; historias estas que brindan respuesta a la blancura silenciosa y devorante del conocimiento normativo y de la teoría (Mirza, 2009).

Para Aura, la educación significa la posibilidad de revertir el daño causado por la colonización; ella proyecta en sí misma la posibilidad de "superar" ese daño que aún se refleja en las aulas. Otras mujeres rarámuri ponen especial énfasis en que la educación no es equitativa y es discriminatoria hasta antes de llegar a la universidad, tal y como plantea Simona:

En mi familia me cuentan que las relaciones interétnicas casi no se daban, en las escuelas existía el racismo, los chabochi no querían a los indígenas. Los maestros no les ponían mucha atención a los indígenas y solo los inscribían en la escuela para completar el grupo. En cambio, los indígenas eran personas amables. Yo, por ser indígena, en la escuela sí fui discriminada. Ya en la universidad no tuve tanta discriminación por parte de mis compañeros, y los maestros nos apoyan con cosas que no conocemos por venir de la sierra. (Simona, Chihuahua, enero de 2015)

Cabe recordar que, según Ogbu y Simons (1998), las minorías solo confían en el profesorado cuando éste muestra un interés sincero y de corazón, que termina por fortalecer la autoestima de los estudiantes. Por lo tanto, el profesorado tiene que mostrar claramente al estudiantado que su cultura es digna de respeto y declarar los estándares que esperan que este estudiantado cumpla. Por ejemplo, Estrella acudió a Salvador, su profesor y tutor, y le comentó que se sentía excluida por sus compañeras en su equipo de trabajo, pues quedaron de avisarle cuando se reunieran a trabajar, pero lo hicieron sin ella, y aunque incluyeron su nombre en los créditos, ella pensó en hablar con cada docente para solicitar que le asignaran trabajos individuales. Sin embargo, Salvador le aconsejó que no lo hiciera, pues se estaba autoexcluyendo; más 
bien le pidió que fuera ella quien repartiera el trabajo y "tomara la batuta". Parece ser que la confianza y las altas expectativas del profesor Salvador en Estrella resultaron fructíferas en el empoderamiento de la joven como estudiante en su propia clase, pues en otra ocasión, cuando uno de los profesores tuvo un enfrentamiento con el grupo académico al que pertenecía Estrella, el alumnado (mestizo y rarámuri) guardó silencio ante las acusaciones injustas del profesor acerca del rendimiento académico del grupo, y Estrella, que normalmente era la alumna más tímida del grupo, alzó la voz y le reclamó al profesor sus inasistencias y su impuntualidad, pues ella es una de las estudiantes más puntuales y responsables.

Por otro lado, Estrella y otras estudiantes rarámuri deben enfrentar algunos dilemas que Ogbu y Simons (1998) identifican en estudiantes de la minoría involuntaria: (1) cumplir con las exigencias escolares y obtener las recompensas por seguir ciertos comportamientos y actitudes de los "blancos"; (2) concordar con las interpretaciones de la comunidad y la desaprobación o ambivalencia hacia las actitudes y comportamientos de la mayoría dominante, pues la comunidad rarámuri también desconfía de la universidad como institución dominante, aunque por otro lado cree que la educación es importante. Así lo vemos expresado en las declaraciones de Catalina, una anciana rarámuri gobernadora en una de las comunidades más grandes de la Sierra Tarahumara:

Solamente teniendo educación podemos avanzar sin tanta dificultad. Creo que
si los jóvenes que están en la universidad guardan nuestra cultura, está bien que
estudien. Los que se quedan en la Sierra siguen cuidando nuestras tradiciones. De
los que van a la universidad hay algunos que tienen cambios muy bruscos; cuando
vuelven a visitar la sierra, ellos quieren saber más que los abuelos y los tíos, pero
aunque hayan estudiado tienen que ir a la sierra y reconocer que sus abuelos saben
más que ellos, porque son sus raíces y deben de escuchar a los ancianos para que los
dirijan por el buen camino. (Catalina, gobernadora en la Sierra Tarahumara; Consulta
para la Reforma de la Ley General de Educación, Chichuahua, noviembre de 2012)

Otro ejemplo de la desaprobación de la comunidad rarámuri hacia las actitudes y comportamientos de la mayoría mestiza - que se sospecha podrían adquirirse en la universidad- es narrado por Alejandra, la única de mis interlocutoras que ya vivía en la ciudad y no había migrado a ella para estudiar. Ella explica que, cuando va de visita a la Sierra Tarahumara, los miembros de la comunidad la discriminan por no poseer los conocimientos que allí son útiles, como los relacionados a la agricultura o actividades domésticas; pero ella está convencida que cuando termine su carrera en Ciencias Agrotecnológicas regresará a su comunidad en la sierra y les dará una "cucharada de su propia medicina" (Entrevista con Alejandra, 11 de septiembre de 2012); es decir, les hará sentir que ellos no tienen los conocimientos con los que ella cuenta.

Lo que Alejandra además expresa es un deseo de romper con los roles de género de su comunidad, que tiene fuertemente demarcada la división sexual del trabajo, ya que cuestionan en ella la ausencia de conocimientos domésticos que son propios de una mujer rarámuri. Ella quiere definir por sí misma qué es lo que debe saber, y crear así su propio conocimiento. Este es uno de los preceptos feministas, crear conocimiento propio.

\section{¿Ser mujer rarámuri universitaria es ser feminista?}

Para las mujeres rarámuri que participaron en mi investigación, ser feminista es "la libertad de la mujer" (Estrella, durante los Talleres de Estudios de Género). Más allá de estas declaraciones, considero que estas mujeres son feministas porque son conscientes de la subordinación que experimentan y desean superarla, tal como reafirma otra de mis interlocutoras: "las mujeres indígenas son muy fuertes, aunque la mayoría son 
dominadas por el hombre. Una mujer indígena es alegre, con ganas de salir adelante, trabajadora, y honesta; sin embargo, es humillada y muy discriminada" (Citlaly, Chihuahua, noviembre de 2014).

Mis interlocutoras no suelen usar el término "feminista" con mucha frecuencia en su vida cotidiana, pero cuando hablan acerca de las personas que inspiran su actuar o se refieren a las personas que admiran, aluden a sus madres e incluso a sí mismas, "por haber llegado lejos enfrentando discriminaciones e injusticias" y lo hacen por ayudar a los suyos, así que van a la universidad para convertirse en alguien que tenga el poder para mejorar sus condiciones en tanto que mujeres.

Dado lo anterior, creo que son feministas, pues el feminismo es la búsqueda concreta emprendida por las mujeres para el bienestar de las mujeres y en diálogo entre sí para destejer los símbolos y las prácticas sociales que las ubican en lugares secundarios. Pues, como afirma la feminista indígena Julieta Paredes: "toda acción organizada por las mujeres indígenas en beneficio de una buena vida para todas las mujeres, se traduce al castellano como feminismo" (cit. en Gargallo, 2014, p. 21).

Cabe anotar que en las mujeres rarámuri perciben un feminismo indígena, muy distinto al feminismo "blanco" que observa a la familia como una atadura, pues ellas se sienten afortunadas de tener a una familia, y creen que es un impulso, tal como podemos percibir en las siguientes afirmaciones: "Mi preocupación más grande es mi familia, su bienestar. Mi posicionamiento como universitaria me está permitiendo brindarles una mejor calidad de vida y economía en un futuro, y les estoy dando orgullo en este momento" (Aura, Chihuahua, noviembre de 2014). Aunque, de igual forma, buscan la independencia de los hombres y dejar atrás episodios de violencia, como declara Samantha:

Me siento afortunada de vivir como a mí me gusta y realizar las cosas a mi manera, y cuando yo quiero y decido. Hasta hoy sigo luchando por dejar atrás los tristes recuerdos que tuve y me siento orgullosa por eso, porque sé que ninguna persona debe empañar mi felicidad. Me preocupa la inseguridad, es algo que ya viví y siento que no estamos protegidas contra la violencia. Una mujer indígena lucha por salir adelante, podemos demostrar que no solo los hombres pueden ser inteligentes o los más fuertes, una mujer cruza las barreras. Estamos aprendiendo que podemos hacer muchas cosas que en algún tiempo se nos decía que no lo lograríamos. Una mujer indígena ya no se deja como antes, trata de salir. (Samantha, Chihuahua, noviembre de 2014)

Y como plantea Verónica:

La mujer indígena quiere aprender nuevas cosas. Visto desde el punto de vista de una mujer joven indígena se cometen muchas injusticias por parte de las personas de dentro y fuera de la etnia, también por las personas mayores que tienen muchas costumbres muy feas, podría decirse que hacen sumisas a sus esposas, entonces las mujeres indígenas jóvenes no queremos eso. (Verónica, Chihuahua, noviembre de 2014)

Estas declaraciones nos recuerdan a las que sustentan el feminismo indígena, en el que las mujeres rechazan los usos y costumbres que atentan contra su dignidad, aunque sean los propios de su grupo (Hernández, 2008; Gargallo, 2014). Cabe anotar que los hombres indígenas pueden reproducir las relaciones de dominación de los hombres blancos, pero el racismo determina que no tengan el mismo poder que estos últimos, debido a las relaciones sociales interétnicas que se constituyen de forma jerárquica. Por ello, las mujeres indígenas no enfatizan únicamente en el patriarcado como un 
problema de urgente solución, al igual que lo hacen las mujeres negras, ${ }^{4}$ sino que $\quad$ 4. Véase Carby (2012). buscan redefinir el término.

\section{Conclusiones}

Las mujeres rarámuri que participan en mi investigación tienen la intención de enorgullecer y ayudar a sus familias y a sí mismas convirtiéndose en universitarias, para ello usan de forma implícita sus saberes étnicos como herramientas que les permiten legitimarse como indígenas y acceder a distintos beneficios otorgados por las instituciones becantes, que tratan a su vez de esencializar su identidad mediante la creación de grupos. No por ello estas mujeres dejan de reflexionar acerca de su formación identitaria; ellas encuentran la forma de levantar su voz para protestar en contra de aquellos actores que no reconozcan su esfuerzo; es decir, seleccionan cuidadosamente qué expectativas cumplir.

Es verdad que, en un afán de satisfacer los deseos de sus familias - particularmente de sus madres-, algunas de ellas pueden acceder a carreras que no deseaban, y esto marca fuertemente su identidad, al definirse como las mujeres que no desean ser. También sucede que, al igual que sus comunidades, suelen desconfiar de las instituciones educativas y la forma en que estas inciden en su identidad. Pero saben que acceder a ellas es la única forma de "superarse" en tanto que están adquiriendo los conocimientos del grupo hegemónico y recuperando los de su grupo de origen para reconstruirlos.

Parte de las políticas educativas dictadas por las instituciones que brindan apoyo a estas mujeres van encaminadas a tratar de empoderarlas para que cambien la cultura de sus comunidades de origen. Las mujeres rarámuri las aceptan, debido a que les ayudan, hasta cierto punto, a superar los usos y costumbres que ellas como mujeres no aprueban al interior de sus comunidades, $\mathrm{y}$ a transformar también las relaciones jerárquicas que se dan a través del contacto interétnico. Estos deseos de "superación" y de "transformación de relaciones jerárquicas" determinan en gran medida un pensamiento feminista.

\section{Financiamiento}

Becaria del Fondo Nacional para la Cultura y las Artes (FONCA) mediante el Programa de Becas para Estudios en el Extranjero 2011-2013. Fondo Nacional para la Cultura y las Artes (FONCA). Programa de Becas para Estudios en el Extranjero 2011-2013. México. 


\section{Q Referencias bibliográficas}

"Abajo, J. y Carrasco, S. (Eds.). (2004). Experiencias de trayectoria y éxito escolar de gitanas y gitanos en España: Encrucijadas sobre educación, género y cambio cultural. Madrid: CIDE/ Instituto de la Mujer.

»Abajo, J. y Carrasco, S. (2011). La situación escolar del alumnado de minorías étnicas: el modelo explicativo ecológico-cultural de John Ogbu. RECERCA, 11, 71-92.

» Brubaker, R. (2002). Ethnicity without groups. Arch Europe Social. XLIII(2), 163-189.

"Carby, H. (2012). Mujeres blancas, iescuchad! El feminismo negro y los límites de la hermandad femenina. En M. Jabardo (Ed.), Feminismos negros (pp. 209-243). Madrid: Traficante de Sueños.

»Castells, M. (2001) La era de la información. Economía, sociedad y cultura. Volumen II: El poder de la identidad. México: Siglo XXI.

»Chávez, M. (2008). Ser indígena en la educación superior, ¿desventajas reales o asignadas? Revista de la Educación Superior, 4(148), 31-55.

»Dietz, G. y Mateos, L. (2011). Interculturalidad y educación intercultural en México. Un análisis de los discursos nacionales e internacionales en su impacto en los modelos educativos mexicanos. México: Secretaría de Educación Pública. Coordinación General de Educación Intercultural y Bilingüe.

»Durin, S. y García, D. (2011). Etnicidad y educación superior: indígenas universitarios en Monterrey. En J. Olvera y B. Vásquez (Coords.), Procesos comunicativos en la migración: de la escuela a la feria popular (pp. 59-84). México: El Colegio de la Frontera Norte/Tecnológico de Monterrey

»Eudine, V.(2011). Aportaciones del feminismo negro al pensamiento feminista: una perspectiva caribeña (Traducción de O. Abasolo). Boletín ECOS, 14 (marzo-mayo). Recuperado de: http://www.fuhem.es/media/ecosocial/file/Boletin\%2oECOS/Bolet\%C3\%ADn\%20 14/Aportaciones\%2odel\%2ofeminismo\%2onegro_V.E.\%20BARRITEAU.pdf

» García, A. (2010). Indígenas universitarios en chihuahua: a la búsqueda de un derecho negado, Tesis de Licenciatura. Escuela Nacional de Antropología e Historia, Unidad Chihuahua, México.

"Gargallo, F. (2014). Ideas y proposiciones de las mujeres de 607 pueblos en nuestra América. México: Corte y Confección.

» Gil, A. 2005. Tejiendo la vida universitaria en la capital: nuevos dilemas de la mujer indígena contemporánea, Tesis de Magíster. Universidad de Los Andes. Bogotá, Colombia

» González, N. 2015. Mujeres indígenas rarámuri universitarias: su resistencia a la opresión. Cadernos de Campo, 24(24), 223-243.

» Hernández, R. (2008). Feminismos poscoloniales: Reflexiones desde el sur del río Bravo. En L. Suárez Navaz y R. Hernández (Eds.), Descolonizando el feminismo: teorías y prácticas desde los márgenes (pp. 68-111). Madrid: Cátedra.

»Lagarde, M. (2011). Los cautiverios de las mujeres: madresposas, monja, putas, presas y locas. México: Universidad Autónoma de México.

» Martín, A. (2008). Antropología del género: culturas, mitos y estereotipos sexuales. Madrid: Cátedra, Universitat de Valencia e Instituto de la Mujer. 
» Mirza, H. (2009). Plotting a history: Black and postcolonial feminisms in "new times". Race Ethnicity and Education, 12(1), 1-10.

» Moore, H. (2009). Antropología y feminismo. Madrid: Cátedra.

» Ogbu, J. y Simons, H. (1998). Voluntary and Involuntary Minorities: A Cultural-Ecological Theory of School Performance with Some Implications for Education. Anthropology \& Education Quarterly, 29 (2), 155-188.

» Programa de Apoyo a Estudiantes Indígenas-PAEl. (2010). Programa de Apoyo a Estudiantes Indígenas. Chihuahua: Universidad Autónoma de Chihuahua.

"Ponferrada, M. (2007). Chicas y poder en la escuela. Identidades académicas, sociales y de género entre jóvenes de la periferia, Tesis de Doctorado. Departamento de Antropología Social y Cultural, Facultad de Filosofía y Letras, Universidad Autónoma de Barcelona, Barcelona, España. Recuperado de http://www.tesisenred.net/bitstream/handle/10803/48705/mpa1de1.pdf?sequence $=1$

»Ponferrada, M. (2012). Género y fronteras étnicas en las vidas escolares, familiares y sociales de niñas y jóvenes de origen extranjero en Cataluña. En C. Blanco (Coord.). VII Congreso Migraciones Internacionales en España, movilidad humana y diversidad Social. Bilbao, España. Universidad del País Vasco (UPV/EHU).

\section{Otras fuentes consultadas}

»Rivas, V. (6 de abril de 2015). De la Sierra Tarahumara, a Universidad de Brasil. El Heraldo de Chihuahua. Recuperado de: http://www.oem.com.mx/elheraldodechihuahua/notas/ n3762816.htm 
